\title{
Kidney Exchange: Further Utilization of Donors via Listed Exchange
}

\author{
Özgür Yılmaz *†
}

September 2013

\begin{abstract}
There is a set of incompatible patient-donor pairs and these pairs are matched pairwise. A match between two pairs corresponds to a paired kidney donation, where pairs exchange donated kidneys, or a paired listed exchange, where the first donor donates a kidney to the deceased donor wait-list, the first patient receives the kidney of the second donor, and the second patient receives a priority on the wait-list. We characterize the set of exchanges with the maximum number of transplants from the set of pairs. This characterization generalizes the well-known Gallai-Edmonds Decomposition Theorem.
\end{abstract}

Keywords : Kidney exchange, Gallai-Edmonds Decomposition

Journal of Economic Literature Classification Numbers: C71, C78, D71, DF8

\footnotetext{
${ }^{*}$ College of Administrative Science and Economics, Koç University, Sarıyer, İstanbul, Turkey 34450. E-mail address: ozyilmaz@ku.edu.tr; Phone: +90 212338 1627; Fax: +90 2123381653.

${ }^{\dagger}$ I would like to thank Tayfun Sönmez and Utku Ünver for helpful comments and conversations. I also would like to thank an associate editor of the journal as well as two anonymous referees for useful suggestions. This research has been supported by TÜBİTAK Grant No. 1001-107K241.
} 


\section{Introduction}

Transplantation is the preferred treatment for the most serious forms of kidney disease. While there is a considerable shortage of deceased donor kidneys, ${ }^{1}$ they are not the only sources for transplantation and it is also possible for a kidney patient to receive a live donor transplant. ${ }^{2}$ We characterize the exchanges utilizing these sources of kidneys such that the number of live donor transplants is maximum.

Medical authorities have developed different programs to increase the number of transplantations. One of these programs is a paired kidney donation. A paired kidney donation involves two incompatible patient-donor pairs, for each of whom a transplant from donor to intended recipient is not possible due to medical incompatibilities, but such that the patient in each pair could receive a transplant from the donor in the other pair. ${ }^{3}$ These two pairs can then exchange donated kidneys.

Another possibility is a listed exchange (also known as living-donor-cadaver-donor exchange), where the recipient of an incompatible pair receives priority on the deceased donor list for providing the wait-list with the kidney from his intended live donor. ${ }^{4}$ Listed exchange can be extended to include two pairs: in a paired listed exchange, there are two donor-patient pairs; the first donor provides a kidney to a candidate on the deceased donor list, the first patient receives the kidney of the second donor, and the second patient receives a priority on the waitlist. ${ }^{5}$ This improves the welfare of the first patient, but also of the second patient, compared to having a long wait for a deceased donor kidney, and it benefits the recipient of the live donor kidney on the wait-list who benefit from the increase in the kidney supply due to an additional live donor.

\footnotetext{
${ }^{1}$ As of October 24, 2012, there are 94,074 patients waiting for kidney transplants in the U.S., with the median waiting time of over 3 years, and in 2011, there were only 11,043 transplants of deceased donor kidneys.

${ }^{2}$ In 2011, there were 5,770 transplants of live donor kidneys.

${ }^{3}$ See Rapaport [15], Ross et al. [16], Ross and Woodle [17], Delmonico [3], Delmonico et al. [4], Segev et al. $[23,24]$.

${ }^{4}$ See Delmonico [3], Kaplan et al. [9], Zenios et al. [28].

${ }^{5}$ Listed exchange is also known as list paired donation. To avoid confusion, we choose to refer to an exchange with two incompatible pairs as paired listed exchange.
} 
While the transplantation community approved the use of the paired kidney donations and listed exchanges to increase kidney donations, it has provided little guidance about how to organize such exchanges. Recently, it is proposed to model kidney exchange as a mechanism design problem. ${ }^{6}$ This approach turns out to be very successful and it is supported by the medical community. Since then, centralized mechanisms based on the paired exchange protocols have been used in the regional exchange programs; in particular, the New England Paired Kidney Exchange Program (NEPKE) has been in practice since 2006, which relies on the design proposed by Roth et al. [19, 20]. The main objective of these kidney exchange programs has been to maximize the number of transplants and we focus on this particular objective.

Kidney exchange has three main characteristics. The first one is a logistical constraint: for any kidney exchange including multiple transplantations, these transplantations are to be carried out simultaneously to avoid conflicts which may arise when a donor gives up after her patient receives a kidney transplant from another donor. This practice excludes the exchanges with three or more incompatible pairs and the only available procedures are paired kidney donation and (paired) listed exchange. The second one is with regards to the preferences of the patients. Due to a medical fact, for a patient, the difference between two live donor kidneys, which are compatible with him, is negligible in terms of the success rate and graft survival rate. Thus, patients are considered to be indifferent between any two compatible live donor kidneys; patients' preferences on the set of compatible live donor kidneys are dichotomous. ${ }^{7}$ The third one is due to an important difference between a paired kidney donation and listed exchange. The former includes two transplantations from live donors and the latter includes a transplantation from a deceased donor. Since both the success rate and graft survival rate are higher for a transplantation from a live donor compared to the one from a deceased donor, ethical concerns arise regarding listed exchange. Despite these concerns, there are cases where listed exchange offers more opportunities in terms of the utilization of the donors, particularly

\footnotetext{
${ }^{6}$ See Roth et al. [18, 19, 20, 21, 22].

${ }^{7}$ Although this view on the patients' preferences for the live donor kidneys is adopted by the transplantation centers, there is a different view arguing that the quality of the match differs across different live donors. See Nicolò and Rodriguez-Álvarez [12] for a discussion of this point.
} 
when there are small groups of patients (Gentry et al. [8]).

Given these characteristics of kidney exchange, the current practice includes a set of incompatible pairs registered for a paired kidney donation and a set of incompatible pairs registered for a listed exchange, and these two sets are treated separately. A simple idea is to combine these two sets to further utilize the donors, in accordance with the kidney exchange programs' current objective of maximizing the number of transplants. Based on this idea, we propose a model where there are two sets of pairs: (i) pairs with a patient expecting a transplantation only from a live donor (these belong to the pool of paired kidney donations) and (ii) pairs with a patient expecting either a transplantation from a live donor or an urgent transplantation from a deceased donor (these belong to the pool of listed exchanges). These (separately formed) pools of pairs are then reconsidered together and combined to further improve the utilization of the donors. The incompatible pairs are matched pairwise according to the medical compatibilities between them. There are two mutually exclusive paired exchanges when two pairs are matched: It is either a paired kidney donation, where the pairs exchange the live donors, or a paired listed exchange, where the first patient receives a live donor kidney and the second pair receives a priority on the wait-list. The interpretation of a paired listed exchange is twofold. First, from an institutional perspective, there is no difference between a listed exchange and a paired listed exchange as far as the patients in the wait-list are concerned. Listed exchange replaces the top priority patient with another patient, thanks to a live donor (the donor of the second patient). As far as the wait-list is concerned, the paired listed exchange does the same thing. For this reason, donation chains of large sizes are possible in practice and in a paired listed exchange, only two pairs take part. Second, the second pair in a paired listed exchange is already registered for a listed exchange and a paired listed exchange does not improve the welfare of the patient in that pair but it helps some other patient receiving a live donor kidney. Thus, while a pair joins the exchange pool with the expectation of getting a transplantation from a live donor; if this does not happen, ex post, paired listed exchange has a flavor of altruism: since the patient in the second pair would have received priority on the wait-list anyways by engaging 
in a listed exchange by itself, only the first pair benefits from this match. ${ }^{8}$ Thus, while two patients benefit from a paired kidney donation, only one patient benefits from a paired listed exchange. Also, there is no issue of strategic manipulation: since we take the type of a patient as given by the current pool that he belongs to, there is no gaming among the pairs in the form of revealing whether to accept priority in the wait-list (in exchange for the donor donating to a patient on the wait-list) or to accept a transplantation only from a live donor. Thus, our approach to this matching problem excludes any type of strategic manipulation per se. We provide a characterization of the matchings with the maximum number of patients receiving a live donor kidney transplantation. Our result generalizes the well-known Gallai [6, 7]-Edmonds [5] Decomposition Theorem, which characterizes the maximum cardinality matchings for the problems where each pairwise match corresponds to a uniform weight.

For the constrained kidney exchange problem, in which only the paired kidney donations are allowed, an efficient and strategy-proof mechanism exists under the dichotomous preferences of the patients and the characterization of the efficient matchings (Roth et al. [19]) is given by the Gallai [6, 7]-Edmonds [5] Decomposition Theorem. There exists also a stochastic mechanism motivated by the fairness considerations. The existence and characterization of the egalitarian matchings (Roth et al. [19]) generalize the corresponding results on the two-sided matching problem (Bogomolnaia and Moulin [2]) to the general matching problem motivated by the kidney exchange. ${ }^{9}$

A new approach is to incorporate compatible pairs into exchange (Sönmez and Ünver [25]); the paired kidney donations between the incompatible pairs as well as between an incompat-

\footnotetext{
${ }^{8}$ In any matching, any unmatched pair including a patient from the set of patients (ii), engages in a listed exchange, thus that patient receives priority in the wait-list as well. These type of unmatched pairs and the pairs matched via a paired listed exchange go to the top of the wait-list together. Since they were already registered for a listed exchange, there is a clear way of prioritizing these pairs. Moreover, although one might think that to be at the top or at the bottom of this short list of patients (who are lined up for a listed exchange) should make a big difference, actually it is not the case. Given the very long waiting times for a cadaveric kidney and that the number of these patients (up for listed exchange) is so small compared to the huge number of patients in the very long wait-list, the difference between being at the top and at the bottom is negligible.

${ }^{9}$ Also, for a preference profile constructed according to the blood-type compatibilities, three-way kidney donations as well as paired kidney donations will have a substantial effect on the number of transplants and larger than three-way kidney donations have less impact Roth et al. [22].
} 
ible pair and a compatible pair are considered. For this problem, the efficient matchings are characterized by extending the Gallai-Edmonds Decomposition Theorem (Sönmez and Ünver $[25]) .{ }^{10}$ In a related work, the matching rules are analyzed such that they provide incentives to patients with compatible donors to enroll in such programs by offering them the chance of receiving an organ with higher expected graft survival (Nicolò and Rodriguez-Álvarez [13]).

For the unconstrained kidney exchange problem, the organization of kidney exchange (under the assumptions of dichotomous preferences of the patients on the live donor kidneys and that the success rates of transplants from live donors are higher than those from cadavers) is explored and the set of efficient and fair random matchings is characterized (Yllmaz [27]). This model is essentially different than the current one in the sense that the former considers the setting where there is no restriction on the number of pairs included in an exchange. ${ }^{11}$ Also, dynamically optimal kidney exchange mechanisms are characterized in a setting where patient-donor pool is evolving dynamically (Ünver [26]).

\section{The model}

A pair consists of a patient and a donor such that the donor cannot medically donate her kidney to the patient of the pair. Let $N$ be the set of all pairs. Given two pairs $x, y \in N$, if the donor of $y$ can medically donate her kidney to the patient of $x$, we say that $x$ is compatible with $y$. Given two pairs, if the donor of each pair can medically donate her kidney to the patient of the other pair, we say that these two pairs are mutually compatible. Each pair $x$ has the following preferences on $N$ : it is indifferent between all compatible pairs, indifferent between all incompatible pairs and it strictly prefers a compatible pair to remaining unmatched and remaining unmatched to an incompatible pair.

A pair can be matched to another pair or remain unmatched. A paired kidney donation (PKD) involves two mutually compatible pairs $x$ and $y$ such that the patient of $x$ receives the

\footnotetext{
${ }^{10}$ This work is closest to the current one and we discuss it further in Section 4.

${ }^{11}$ Roth et al. $[18,22]$ also analyze the unconstrained problem.
} 
kidney of the donor of $y$ and the patient of $y$ receives the kidney of the donor of $x$. A paired listed exchange (PLE) involves two pairs $x$ and $y$, such that $x$ is compatible with $y$ and $y$ is not compatible with $x$, the patient of $x$ receives the kidney of the donor of $y$, the donor of $x$ donates to a patient in the wait-list, and the patient of $y$ receives priority in the wait-list. Due to a medical fact, that a live donor kidney has a substantially higher patient survival and graft survival rates than the deceased donor kidney, each pair strictly prefers a compatible pair to a deceased donor kidney.

There are two types of pairs: a p-pair prefers being unmatched to a deceased donor kidney transplantation and an l-pair prefers the highest priority on the wait-list to being unmatched. The sets of p-pairs and the l-pairs are denoted by $N_{p}$ and $N_{l}$, respectively and they partition the set $N$.

A feasible exchange matrix $R=\left[r_{x, y}\right]_{x, y \in N}$ identifies all feasible exchanges where

$$
r_{x, y}= \begin{cases}2 & \text { if } y \in N \backslash\{x\}, \text { and } x, y \text { are mutually compatible } \\ 1 & \text { if } x \text { is compatible with } y, y \text { is not compatible with } x \text { and } y \in N_{l} \\ 0 & \text { otherwise. }\end{cases}
$$

A pair $(x, y)$ is referred to as a feasible paired kidney donation if $r_{x, y}=2$ and as a feasible paired listed exchange if $r_{x, y}=1$.

A kidney exchange problem (or simply a problem) $(N, R)$ consists of a set of pairs and a feasible exchange matrix. Given a problem $(N, R)$ and $N^{\prime} \subseteq N$, the reduced problem is denoted by $\left(N^{\prime},\left.R\right|_{N^{\prime}}\right.$, where $\left.R\right|_{N^{\prime}}$ is the reduced matrix of $R$ on $N^{\prime}$. A matching is a function $\mu: N \rightarrow N$ such that for all $x, y \in N, \mu(x)=y$ if and only if $\mu(y)=x$, and $[\mu(x)=y$ and $x \neq y$ ] imply either $r_{x, y} \neq 0$ or $r_{y, x} \neq 0$, with the convention that $\mu(x)=x$ means $x$ being unmatched at $\mu$. Thus, a matching $\mu$ specifies a set of mutually feasible paired kidney donations and paired listed exchanges. For a matching $\mu$, if $x$ and $y$ are matched via a PLE, we denote this by $\mu_{1}(x)=y$ and if $x$ and $y$ are matched via a PKD, we denote this by $\mu_{2}(x)=y \cdot{ }^{12}$ For a

\footnotetext{
${ }^{12}$ Whenever convenient and no confusion arises, we denote by $\mu$ also the set of pairs matched via the function $\mu$. Since $\mu_{1}$ and $\mu_{2}$ correspond to the set of PLE's and PKD's, respectively, this notation implies $\mu=\mu_{1} \cup \mu_{2}$.
} 
problem $(N, R)$, let $\mathcal{M}(N, R)$ denote the set of all matchings.

Since any pair can always receive priority in the wait-list by simply accepting to be in a listed exchange, when comparing two different matchings, only the patients receiving a live donor kidney transplantation should be taken into consideration. For each matching $\mu$, let $T^{\mu}$ denote the set of all pairs who receive a transplant from a live donor. Formally,

$$
T^{\mu}=\left\{x \in N:(x, y) \in \mu_{1} \cup \mu_{2} \text { for some } y \in N\right\}
$$

For each $\mu, \mu^{\prime} \in \mathcal{M}, \mu$ Pareto-dominates $\mu^{\prime}$ if, each $x \in N$ weakly prefers $\mu(x)$ to $\mu^{\prime}(x)$, and some $x \in N$ strictly prefers $\mu(x)$ to $\mu^{\prime}(x)$. A matching $\mu \in \mathcal{M}$ is Pareto efficient if no other matching Pareto dominates $\mu$. For a problem $(N, R)$, let $\mathcal{E}(N, R)$ denote the set of Pareto efficient matchings.

\section{Maximum and p-maximum matchings}

Our model relies on the interpretation of the sets $N_{p}$ and $N_{l}$. There is a set of pairs $N_{p}$, each of whom expects to receive a transplant from a live donor and there is a set of pairs $N_{l}$, each of whom has accepted to be in a listed exchange to receive priority in the wait-list. These two sets are integrated to improve social welfare via the extended set of feasible PKD's and PLE's. For example, let $N_{p}=\{x\}$ and $N_{l}=\{y\}$ such that $r_{x, y}=1$. If these two pairs are considered separately, then the pair $x$ remains unmatched, and the pair $y$ receives priority in the wait-list. On the other hand, if these two sets are considered together as suggested in our model, then the pair $x$ receives a transplant from the donor of $y$ and the pair $y$ receives priority in the wait-list.

When there are no l-pairs, a well-known result, the Gallai [6, 7]-Edmonds [5] Decomposition Theorem, ${ }^{13}$ characterizes the structure of Pareto efficient matchings and the same number of

Also, for a matching $\mu,(x, y) \in \mu_{2}$ means that the patient of each pair receives a kidney from the donor of the other pair and $(x, y) \in \mu_{1}$ means that the patient of $x$ receives a kidney from the donor of $y$, the donor of $x$ donates to a patient on the wait-list, the patient of $y$ receives priority in the wait-list, and $y \in N_{l}$. Thus, for $x \neq y,(x, y) \in \mu_{2}$ implies $(y, x) \in \mu_{2}$, and $(x, y) \in \mu_{1}$ implies $(y, x) \notin \mu_{1}$.

${ }^{13}$ See Section 5.3. for the formal statement of this result. 
pairs are matched at each Pareto efficient matching. ${ }^{14}$ However, for a kidney exchange model with p-pairs and l-pairs, the number of pairs, who receive a transplant from a live donor, may be different in different Pareto efficient matchings.

Example 1: Let $N_{p}=\{x\}, N_{l}=\{y, z\}$ and the feasible exchange matrix $R$ be such that $r_{x, y}=1$ and $r_{y, z}=2$. There are two Pareto efficient matchings, $\mu=\{(x, y)\}$ and $\mu^{\prime}=\{(y, z)\}$, where $\left|T^{\mu}\right|=1$, and $\left|T^{\mu^{\prime}}\right|=2$. Also, the number of p-pairs, who receive a transplant from a live donor, is different in different Pareto efficient matchings.

Thus, some Pareto efficient matchings can be improved in terms of the number of pairs who receive a transplant from a live donor. Actually, the opportunity of increasing the number of transplants in general forms the main objective of the transplantation centers, they aim at the arrangements of the exchanges such that the number of transplants is maximized. We focus on this particular objective as well. A matching $\mu$ is maximum (or has the maximum number of transplants form live donors) if there is no other matching $\mu^{\prime}$ such that $\left|T^{\mu^{\prime}}\right|>\left|T^{\mu}\right|$. Also, the transplantation centers' preferred exchange is the PKD. Thus, it is plausible to minimize the number of the PLE's, while maximizing the number of transplants. A matching is called p-maximum if it is maximum and it has the maximum number of PKD's in the set of the maximum matchings. Given a problem $(N, R)$, let $\mathcal{E}^{m}(N, R)$ denote the set of p-maximum matchings. ${ }^{15}$

The existing exchange mechanism used in practice considers the pool of the p-pairs separately and matches the pairs in such a way that the number of transplants is maximized. Focusing on the same objective, our approach considers the sets of the l-pairs and p-pairs together, which are originally formed separately. The l-pairs are located possibly in several regions which are geographically close to the location of the pool of the p-pairs. These l-pairs are then integrated to the pool of the p-pairs so that the number of transplants is further en-

\footnotetext{
${ }^{14}$ Observe that when there no l-pairs, only the PKD's are feasible, and there is no PLE in a matching.

${ }^{15} \mathrm{~A}$ p-maximum matching always exists. Since the problem is finite, a maximum matching exists and a pmaximum matching is maximum such that the number of PKD's is maximum among these maximum matchings, which clearly exist, again by finiteness of the problem.
} 
hanced. ${ }^{16}$ It is clear that each l-pair is weakly better off after the integration since such a pair $x$ is already registered for a listed exchange to receive priority in the wait-list, and after the integration, pair $x$ will receive either a live donor kidney transplant (if $(x, y) \in \mu$ ) or priority in the wait-list (if $x$ is unmatched or $(y, x) \in \mu_{1}$, in either case $x$ is back in a (paired) listed exchange). The question is whether the welfare of the p-pairs (weakly) improves as well, in other words, whether the l-pairs do not have a negative externality on the welfare of the p-pairs, if the transplantation center insists on the p-maximum matchings. More specifically, suppose a maximum (equivalently, p-maximum in this particular setting of only p-pairs) matching is fixed for the set of p-pairs only. Let $T$ be the set of pairs who receive a transplant in this matching. Then, after the integration of the l-pairs to the pool of p-pairs, does there exist a p-maximum matching in this new problem, so that all the patients in $T$ receive a live donor kidney transplant? As our first result shows, the answer is positive.

Proposition 1 Let $\left(N_{p} \cup N_{l}, R\right)$ be a problem. Let $\mu \in \mathcal{E}^{m}\left(N_{p},\left.R\right|_{N_{p}}\right)$. Then, there exists a matching $\mu^{\prime} \in \mathcal{E}^{m}\left(N_{p} \cup N_{l}, R\right)$ such that $T^{\mu^{\prime}} \supseteq T^{\mu}$.

This result implies that the efficiency gains from the integration of the l-pairs to the pool of p-pairs are always at the full level, even if the set of matchings is restricted so that each pair in a subset of $N_{p}$ is guaranteed to receive a live donor kidney transplant. ${ }^{17}$ Thus, given a priority ordering on $N_{p}$ and a priority mechanism based on this ordering ${ }^{18}$, a p-maximum matching can

\footnotetext{
${ }^{16}$ We abstract the logistical issues regarding how to bring a patient and a donor from different regions together. A recent work analyzes ways of overcoming geographical disparities (Ata et al. [1]).

${ }^{17}$ This proposition and the discussion before demonstrate that the integration of the sets of p-pairs and l-pairs makes each pair weakly better off. This result relies on the characteristics of our model and the way the sets of p-pairs and l-pairs are interpreted. It clearly does not hold if we interpret the integration in a different way if, for example, we consider the welfare of the patients in a pool where only PLE is possible initially and then PKD becomes available (although this is far from being a suitable way of modeling kidney exchange since its characteristics and the current practice exclude situations where PLE is available but PKD is not). This extension of the possible exchange procedures may hurt the patients who engage in a PLE before the change. For example, for the set of pairs $N=\{x, y, u, v\}$ and the feasible exchange matrix $R$ with $r_{x, y}=r_{u, v}=1$, $r_{y, v}=2$, the only p-maximum matching when only PLE is possible, is $\mu=\{(x, y),(u, v)\}$. If PKD becomes available as well, the only p-maximum matching is $\mu^{\prime}=\{(y, v)\}$ where pairs $x$ and $u$ are adversely affected by the extension.

${ }^{18} \mathrm{~A}$ priority mechanism matches as many patients as possible (among the matchings with the maximum possible number of patients matched in a paired kidney donation) starting with the patient with the highest priority and following the priority ordering.
} 
be obtained under the integration without making a pair in $N_{p}$ worse off with respect to the outcome of this priority mechanism.

\section{The structure of p-maximum matchings}

Given the sets $N_{p}$ and $N_{l}$, our goal is to characterize the structure of p-maximum matchings. As discussed in the introduction, there is no room for strategic manipulation because our model relies on the fact that the types of the patients are already revealed and the sets $N_{p}$ and $N_{l}$ are known and they are combined together to further improve the utilization of the donors. Thus, we focus only on the efficiency issues. For each problem $(N, R)$, let

$$
D(N, R)=\left\{x \in N: \exists \mu \in \mathcal{E}^{m}(N, R) \text { s.t. } \mu(x)=x\right\}
$$

Let $A_{1}(N, R)$ be the set of pairs who are part of only PLE's in each p-maximum matching and have a compatibility with at least one pair in $D(N, R)$.

$$
A_{1}(N, R)=\left\{x \in N: \forall \mu \in \mathcal{E}^{m}(N, R), \mu_{1}(x) \neq x \text { and } \exists y \in D(N, R) \text { s.t. }\left\{r_{x, y}, r_{y, x}\right\} \neq\{0\}\right\} \text {. }
$$

A component $C$ of $N^{\prime} \subseteq N$ is such that (i) for each $x \in C$ and $y \in N^{\prime} \backslash C, x$ is not compatible with $y$ and $y$ is not compatible with $x$, (ii) for each $C^{\prime} \subsetneq C$, there exist $u \in C^{\prime}$ and $v \in N^{\prime} \backslash C^{\prime}$ such that either $u$ is compatible with $v$ or $v$ is compatible with $u$.

Theorem 1 Let $(N, R)$ be a problem. Then,

1. in any p-maximum matching, each pair in $A_{1}(N, R)$ is matched to a pair in $D(N, R)$;

2. if the set $N \backslash A_{1}(N, R)$ contains a component $C$ in $D(N, R)$, then

(a) for each $x \in C$, the problem $\left(C \backslash\{x\},\left.R\right|_{C \backslash\{x\}}\right)$ has a matching with each pair being matched,

(b) in any p-maximum matching, each pair in $C$ but one is matched to a pair in $C$, 
(c) in any p-maximum matching, at most one pair in $C$ is matched to a pair in $N \backslash C$ and such a pair, if any, is matched to a pair in $A_{1}(N, R)$.

To illustrate the idea and the sets $D(N, R)$ and $A_{1}(N, R)$, we provide a simple example.

Example 2: Let $N_{p}=\{u, v\}, N_{l}=\{x, y, z\}$ and the feasible exchange matrix $R$ be as follows:

$\begin{array}{llllll} & x & y & z & u & v \\ x & 0 & 1 & 1 & 0 & 0 \\ y & 0 & 0 & 2 & 0 & 0 \\ z & 0 & 2 & 0 & 2 & 0 \\ u & 1 & 1 & 2 & 0 & 0 \\ v & 1 & 0 & 1 & 0 & 0\end{array}$

In any p-maximum matching, three patients receive a transplantation from a live donor; also, $z$ is matched to either $u$ or $y$ via a PKD, and $x$ is matched to either $u, y$ or $v$ via a PLE. There are four such p-maximum matchings, each of which is such that one of the pairs $u, v$ and $y$ is unmatched. Thus, $D(N, R)=\{u, v, y\}$ and $A_{1}(N, R)=\{x\}$.

Our result conveys the following: For our general model of kidney exchange, while the Gallai-Edmonds Decomposition (GED) $)^{19}$ structure is not fully present anymore, a restricted GED-type structure with a similar economic interpretation (as in the pairwise kidney exchange with only PKD's) still exists. There exists a set of pairs each of which will always engage in a PLE with a pair from another given set; also, if a subset of the former set corresponds to a particular component structure in the reduced problem, then the structure of matchings of the pairs in that component is equivalent to the one implied by the GED-type structure.

Our result extends the GED Theorem: given $(N, R)$, when no two pairs are mutually compatible, thus, when for all $x, y \in N, r_{x, y} \in\{0,1\}$, the structure given in Theorem 1 reduces to the GED Theorem; we discuss further how the latter theorem derives from our result in Section 5.3. Moreover, the characterization given in Theorem 1 is tight and there are examples

\footnotetext{
${ }^{19}$ See Section 5.3 for the the formal statement of the GED Theorem.
} 
which illustrate that there is no further GED-type structure than the one given in Theorem $1 .^{20}$

A similar (and independent) result is obtained by Sönmez and Ünver [25] via an altruistic kidney exchange model introduced by the authors; their model includes the compatible pairs in a way that the PKD's occur between the incompatible pairs or between an incompatible pair and a compatible pair. This can be interpreted as a particular case of our model: Each compatible pair can be matched only with an incompatible pair and if it is matched, it benefits the incompatible pair that it is matched but not itself, because its patient is compatible with its donor and would receive her kidney anyways. Thus, the compatible pair accepts to be matched with an incompatible pair purely for altruistic reasons. Thus, in such a match, only the patient of the incompatible pair benefits. Thus, $N_{l}$ is the set of compatible pairs in this model. However, there is a restriction: no two compatible pairs can be matched. Thus, $x, y \in N_{l}$ implies $r_{x, y}=0 .{ }^{21}$ For this particular class of problems, the GED-type structure is fully preserved. Thus, our result shows the extent to which the GED structure is maintained under a generalization of the altruistic kidney exchange model. ${ }^{22}$

\section{Appendix}

\subsection{Preliminaries on graphs}

Our goal is to characterize the set of p-maximum matchings, thus we focus on the number of patients receiving a transplant from a live donor. This implies that we can represent a kidney exchange problem by a weighted graph, where certain edges stand for a feasible PKD and others for a feasible PLE. We introduce below some basic notions in graph theory to be used in converting a feasible exchange matrix into a graph.

A problem $(N, R)$ can be represented by a weighted graph $G=(V, E, w)$, where $V$ is the

\footnotetext{
${ }^{20}$ These examples are available upon request.

${ }^{21}$ Note that in the general model that we consider, there is no such restriction for the l-pairs: an l-pair can be compatible with another l-pair, also two l-pairs can be mutually compatible.

${ }^{22}$ Another related result, which also builds on the GED Theorem, is related to fractional matchings. We discuss this extension and its relation to our work in Section 5.3.
} 
set of vertices, $E$ is the set of edges, and $w$ is a weight function, $w: E \rightarrow\{1,2\}$. The graph representation of a problem $(N, R)$ is obtained as follows: Each pair $u$ is a vertex, ${ }^{23}$ thus $V=N$. Let $u, v \in V$ be two vertices. If $r_{u, v}=2$, then the set $E$ contains the edge $u v$, the weight of which is 2. If $r_{u, v}=1$, then the set $E$ contains the edge $u v$, the weight of which is 1 .An edge with weight 1 is called a thin edge; an edge with weight 2 is called a thick edge. For a weighted graph $G=(V, E, w)$, and $E^{\prime} \subseteq E$, we call $w\left(E^{\prime}\right)=\sum_{u v \in E^{\prime}} w(u v)$ as the weight of $E^{\prime}$. An edge $u v$ is said to be incident with the vertex $u$ (and the vertex $v$ ). A vertex $u$ is a neighbor of $v$ if the set $E$ contains the edge $u v$. The neighbor set of a set of vertices $V^{\prime} \subseteq V$ is the union of the neighbors of the vertices in $V^{\prime}$. A set of vertices $V^{\prime} \subseteq V$ is a component of $G$ if it is equivalent to its neighbor set and $V^{\prime \prime} \subsetneq V^{\prime}$ implies that $V^{\prime \prime}$ is not equal to the neighbor set of $V^{\prime \prime}$.

Let $G=(V, E, w)$ be a weighted graph. For a vertex set $V^{\prime}$, let $G-V^{\prime}$ denote the subgraph obtained by removing each $v \in V^{\prime}$ and the edges incident with $v$. We denote $G-\{u\}$ by $G-u$. For a subgraph $H$ of $G$, let $H_{2}$ be the subgraph of $H$ which consists of the thick edges in $H$. The set of thin and thick edges are denoted by $E_{1}(G)$ and $E_{2}(G)$, respectively. A matching $\mu$ is a subset of the edges such that no two edges meet at a common vertex. Let $\mathcal{M}(G)$ denote the set of all matchings. Let $\nu(G)=\underset{\mu \in \mathcal{M}(G)}{\operatorname{Max}} w(\mu)$. A matching $\mu$ has maximum weight if $w(\mu)=\nu(G)$. A matching is p-maximum if it has the maximum weight and has the maximum number of thick edges among all maximum weight matchings. A vertex $v$ is free with respect to a matching $\mu$, or the matching $\mu$ misses $v$, if $v$ not incident with any edge in $\mu$. We say that a matching $\mu$ covers a vertex $v$ if $v$ is not free with respect to $\mu$. A subset of the edges $\mu$ is a perfect matching if no vertex is free and a near-perfect matching if only one vertex is free with respect to $\mu$. A graph is called hypomatchable if for each $v \in V$, $G-v$ has a perfect matching. A path is a set of edges $\left\{v_{1} v_{2}, v_{2} v_{3}, \ldots, v_{k-1} v_{k}\right\}$; it is called a cycle if $v_{1}=v_{k}$. A path (or a cycle) is alternating with respect to a matching $\mu$ if its edges are alternately in $\mu$ and not in $\mu$. An augmenting path is an alternating path between

\footnotetext{
${ }^{23}$ For expositional convenience, we use the generic notation $u, v, \ldots$ for the vertices along $x, y, \ldots$ which is used to denote the pairs in Section 2-4.
} 
free vertices. An augmenting path with respect to a matching $\mu$ is called a $\mu$-augmenting path. If the weights of the edges are uniform, then a well-known result in matching theory characterizes the condition for the maximum cardinality of a matching.

Theorem 2 (Berge's Lemma) Given all the edges have the same weight, a matching $\mu$ is maximum if and only if there does not exist a $\mu$-augmenting path.

\subsection{Proofs}

First, we extend Theorem 2 to the graphs consisting of both thick and thin edges. Let $\oplus$ denote the symmetric difference operator, for $E^{\prime}, E^{\prime \prime} \subseteq E, E^{\prime} \oplus E^{\prime \prime}=\left(E^{\prime} \backslash E^{\prime \prime}\right) \cup\left(E^{\prime \prime} \backslash E^{\prime}\right)$.

Lemma $1 A$ matching $\mu$ in a graph $G$ is p-maximum if and only if $\left|\mu_{2}\right|=\nu\left(G_{2}\right)$ and there does not exist a $\mu$-augmenting path $P$ with $E_{2}(P \cap \mu)=E_{2}(P \backslash \mu)$.

Proof. (Only if) Clearly, $\left|\mu_{2}\right| \leq \nu\left(G_{2}\right)$. Suppose the inequality is strict. Then, by Theorem 2, there is a $\mu_{2}$-augmenting path in $G_{2}$, say $P$. If both of the end vertices in $P$ are free in $\mu$, then $w(\mu \oplus P)=w(\mu)+2$, which contradicts $\mu$ having the maximum weight, thus being p-maximum. Suppose only one of the end vertices, say $u$, is covered in $\mu$, say $u x \in \mu$. Since $P$ is a $\mu_{2}$-augmenting path in $G_{2}$, the edge $u x$ is thin. Then, $w(\mu \oplus(P \cup\{u x\}))=w(\mu)+1$, which contradicts $\mu$ having the maximum weight, thus being p-maximum. Suppose both end vertices in $P$, say $u$ and $v$, are covered by the (thin) edges in $G$, say by $u x$ and $v y$. Then, $w(\mu \oplus(P \cup\{u x, v y\}))=w(\mu)$ and the matching $\mu \oplus(P \cup\{u x, v y\})$ has one more thick edge than the matching $\mu$, which contradicts that $\mu$ is p-maximum. Thus, $\left|\mu_{2}\right|=\nu\left(G_{2}\right)$. Now,

suppose there exists a $\mu$-augmenting path $P$ such that $E_{2}(P \cap \mu)=E_{2}(P \backslash \mu)$. Since the end vertices of the path $P$ are free, the set $P \oplus \mu$ is another matching. Moreover, we have $E_{2}(\mu \oplus P)=E_{2}(\mu \backslash P) \cup E_{2}(P \backslash \mu)=E_{2}(\mu \backslash P) \cup E_{2}(P \cap \mu)=E_{2}(\mu)$, where the first equality is by definition of the symmetric difference set, the second by the assumption and the third by the fact that the end vertices of $P$ are free. Thus, both $\mu$ and $P \oplus \mu$ have equal number of thick edges. Since the matching $P \oplus \mu$ contains one more edge than $\mu$ and both $\mu$ and $P \oplus \mu$ have 
equal number of thick edges, $w(P \oplus \mu)=w(\mu)+1$, which contradicts the matching $\mu$ having the maximum weight.

(If) Suppose $\mu$ is a non-maximum weight matching with $\left|\mu_{2}\right|=\nu\left(G_{2}\right)$. Let $\mu^{\prime}$ be a p-maximum matching. Since, in a matching, no two edges meet at a common vertex, each vertex is incident with at most one vertex in $\mu$ and one vertex in $\mu^{\prime}$. Thus, the set $\mu \oplus \mu^{\prime}$ contains connected components of the form of either a $\mu$-alternating path or a $\mu$-alternating cycle. Since $w\left(\mu^{\prime}\right)>$ $w(\mu)$, one of these alternating paths or cycles, say $H$, is such that $w\left(H \cap \mu^{\prime}\right)>w(H \cap \mu)$. Suppose there exists such an alternating path, say $P$. Since $\mu$ contains the maximum possible number of thick edges, $E_{2}(P \cap \mu) \geq E_{2}\left(P \cap \mu^{\prime}\right)$. Otherwise, $\mu \oplus P$ contains more thick edges than $\mu$, which is a contradiction. Now, since $P$ is an alternating path, the difference between the number of edges of $P \cap \mu$ and $P \cap \mu^{\prime}$ can be at most one. Suppose $\left|P \cap \mu^{\prime}\right|=|P \cap \mu|$ or $\left|P \cap \mu^{\prime}\right|=|P \cap \mu|-1$. Since $E_{2}(P \cap \mu) \geq E_{2}\left(P \cap \mu^{\prime}\right)$, in either case, $w\left(P \cap \mu^{\prime}\right) \leq w(P \cap \mu)$. Thus, $P$ is such that $\left|P \cap \mu^{\prime}\right|=|P \cap \mu|+1$ and $E_{2}(P \cap \mu)=E_{2}\left(P \cap \mu^{\prime}\right)$. Note that this implies that $P$ is a $\mu$-augmenting path with $E_{2}(P \cap \mu)=E_{2}(P \backslash \mu)$. Now, suppose there exists an alternating cycle, say $C$, such that $w\left(C \cap \mu^{\prime}\right)>w(C \cap \mu)$. Since each vertex is incident to at most one vertex in $\mu$ and one vertex in $\mu^{\prime}$, the cycle $C$ is an even size cycle. But, since $E_{2}(C \cap \mu) \geq E_{2}\left(C \cap \mu^{\prime}\right)$ implies $w(C \cap \mu) \geq w\left(C \cap \mu^{\prime}\right)$, this is impossible.

\section{PROOF of PROPOSITION 1:}

Let $\left(N_{p} \cup N_{l}, R\right)$ be a problem. Let $\mu \in \mathcal{E}^{m}\left(N_{p},\left.R\right|_{N_{p}}\right)$. Let $\mu^{\prime} \in \mathcal{E}^{m}\left(N_{p} \cup N_{l}, R\right)$ such that $T^{\mu^{\prime}} \nsupseteq T^{\mu}$. Then, there exists $x \in T^{\mu} \backslash T^{\mu^{\prime}}$. Since $x$ is covered by $\mu$ but not by $\mu^{\prime}$, the set $\mu \oplus \mu^{\prime}$ contains a path $P$ starting at $x$. Let $P=\left\{x x_{1}, x_{1} x_{2}, \ldots, x_{k-1} x_{k}, x_{k} y\right\}$ be this path. Observe that since $\mu$ is a matching for the problem $\left(N_{p},\left.R\right|_{N_{p}}\right)$ and all the vertices $x_{1}, x_{2}, \ldots, x_{k}$ are covered by $\mu$ and $\mu^{\prime}$, these vertices are in $N_{p}$. Moreover, according to the definition of $R$, $r_{a, b}=1$ implies that $b \in N_{l}$. Thus, all the edges $x_{1} x_{2}, x_{2} x_{3}, \ldots, x_{k-1} x_{k}$ in $P$ are thick. If $y$ is covered by $\mu$, then $P$ is a $\mu^{\prime}$-augmenting path: the matching $P \oplus \mu^{\prime}$ contains more thick edges and has a higher weight than $\mu^{\prime}$, contradicting that $\mu^{\prime}$ is p-maximum. Thus, $y$ is not covered by $\mu$ but covered by $\mu^{\prime}$. Thus, the path $P$ contains even number of edges. If the edge $x_{k} y$ is thin, 
then, since all other edges in $P$ are thick, the matching $P \oplus \mu^{\prime}$ has a weight equal $w\left(\mu^{\prime}\right)+1$, which contradicts that $\mu^{\prime}$ is p-maximum. Thus, the edge $x_{k} y$ is thick, and the matching $P \oplus \mu^{\prime}$ is p-maximum as well. Observe that the matching $P \oplus \mu^{\prime}$ covers all the vertices $x, x_{1}, x_{2}, \ldots, x_{k}$, but not $y$. Since $y$ is not covered by $\mu$, we obtained a p-maximum matching $P \oplus \mu^{\prime}$ such that $T^{P \oplus \mu^{\prime}}=\left(T^{\mu^{\prime}} \cup\{x\}\right) \backslash\{y\}$. By applying the same argument recursively, a p-maximum matching is obtained, where it covers all the vertices in $T^{\mu}$.

\section{PROOF of THEOREM 1:}

Let $D_{1}(N, R)$ and $D_{2}(N, R)$ be the sets of pairs who are in $D(N, R)$ and part of only PLE's and of only PKD's, respectively, in any p-maximum matching.

Let $D_{1,2}(N, R)=D(N, R) \backslash\left(D_{1}(N, R) \cup D_{2}(N, R)\right)$. Let $C_{1}(N, R)$ be the set of pairs who are part of only PLE's in each p-maximum matching, which are not in $A_{1}(N, R)$.

$$
C_{1}(N, R)=\left\{x \in N \backslash A_{1}(N, R): \forall \mu \in \mathcal{E}^{m}(N, R), x y \in \mu_{1} \text { for some } y\right\}
$$

Let $A_{1,2}(N, R)$ be the set of pairs who are part of a PLE in some p-maximum matchings and part of a PKD in the remaining p-maximum matchings, who have a compatibility with at least one pair in $D_{1}(N, R) \cup D_{1,2}(N, R)$.

Let $C_{1,2}(N, R)$ be the set of pairs who are part of a PLE in some p-maximum matchings and part of a PKD in the remaining p-maximum matchings, who are not in $A_{1,2}(N, R)$.

$C_{1,2}(N, R)=\left\{x \in N \backslash\left(D(N, R) \cup A_{1,2}(N, R)\right): \exists \mu, \mu^{\prime} \in \mathcal{E}^{m}(N, R)\right.$, s.t $x$ is matched in $\left.\mu_{1}, \mu_{2}^{\prime}\right\}$.

A problem $(N, R)$ can be represented by a graph $G$, as explained in Section 5.1, and in what follows, we refer to its graph representation rather than to the problem $(N, R)$ itself. Moreover, the sets $D(N, R)$ and $A_{1}(N, R)$ in Section 4, as well as the sets given in the previous paragraph in this subsection, can be redefined with the $\operatorname{argument} G$ instead of the argument $(N, R)$, where the words "pair", "PLE" and "PKD" are replaced by "vertex", "thin edge" and "thick edge", 
respectively. Thus, for example, we use $D(G)$ instead of $D(N, R)$.

Lemma 2 If $u \in D_{1}(G)$, then each edge incident with $u$ in $G$ is thin.

Proof. Let $u \in D_{1}(G)$. Suppose there exists a thick edge $u v$ in $G$. Since $u \in V\left(G_{2}\right)$, either $u \in D\left(G_{2}\right)$ or $u \in V\left(G_{2}\right) \backslash D\left(G_{2}\right)$. Suppose the latter holds; that is, $u$ is matched in any maximum matching in $G_{2}$. By Lemma 1, each p-maximum matching $\mu$ in $G$ is such that $\left|\mu_{2}\right|=\nu\left(G_{2}\right)$. Thus, $u$ is matched in any p-maximum matching via a thick edge in $G$, which contradicts $u \in D_{1}(G)$. Thus, $u \in D\left(G_{2}\right)$. Let $M$ be a p-maximum matching missing $u$. The vertex $v$ must be covered in $M$, otherwise $M \cup\{u v\}$ is also a matching with a higher weight than $M$, which contradicts $M$ being a p-maximum matching. Let $v w \in M$. If $v w$ is thin, then $(M \backslash\{v w\}) \cup\{u v\}$ has a higher weight than $M$, which contradicts $M$ being p-maximum. Thus, $v w$ is thick. Then, clearly $(M \backslash\{v w\}) \cup\{u v\}$ is another p-maximum matching. But this contradicts $u \in D_{1}(G)$.

Lemma 3 Let $G$ be any graph. Let $u \in A_{1}(G)$. Then,

(i) $D(G-u)=D(G)$ and

(ii) $D_{1}(G-u)=D_{1}(G)$.

Proof. Let $u \in A_{1}(G)$.

(i) Let $v \in D(G-u)$. Let $M$ be a p-maximum matching in $G-u$, which misses $v$. By definition of $A_{1}(G)$, there exists a vertex in $D(G)$, say $v^{\prime}$, which is a neighbor of $u$. Let $M^{\prime}$ be a p-maximum matching in $G$ missing $v^{\prime}$. If $v^{\prime}=v$ or $M^{\prime}$ misses $v$, then either case, there is a p-maximum matching $\left(M^{\prime}\right)$ in $G$, which misses $v$. Thus, suppose $M^{\prime}$ covers $v$. Consider the subgraph $M \oplus M^{\prime}$. Since both $M$ and $M^{\prime}$ are matchings, $M \oplus M^{\prime}$ contains alternating paths and cycles. Since $M$ misses $v, M \oplus M^{\prime}$ contains an alternating path, $P$, starting at $v$ with an edge in $M^{\prime}$.

Suppose $P$ ends with an edge of $M$. Then, since $M$ is in $G-u$, the path $P$ does not cover $u$. If $w(P \cap M)>w\left(P \cap M^{\prime}\right)$, then $M^{\prime} \oplus P$ is another matching in $G$ such that $w\left(M^{\prime} \oplus P\right)>w\left(M^{\prime}\right)$, contradicting that $M^{\prime}$ is p-maximum; similarly, if $w\left(P \cap M^{\prime}\right)>w(P \cap M)$, then it contradicts 
that $M$ is p-maximum. Thus, $w(P \cap M)=w\left(P \cap M^{\prime}\right)$. This, together with the fact that $P$ is an even-length alternating path, implies that $P \cap M$ contains the same number of thick edges as $P \cap M^{\prime}$. But then $M^{\prime} \oplus P$ is another p-maximum matching in $G$, which misses $v$. Thus, $v \in D(G)$.

Suppose $P$ ends with an edge of $M^{\prime}$. We claim $P$ ends at $u$. Suppose not. Then, if $w(P \cap M)>$ $w\left(P \cap M^{\prime}\right)$, then $M^{\prime} \oplus P$ is another matching in $G$ such that $w\left(M^{\prime} \oplus P\right)>w\left(M^{\prime}\right)$, contradicting that $M^{\prime}$ is p-maximum. Similarly, if $w\left(P \cap M^{\prime}\right)>w(P \cap M)$, then it contradicts that $M$ is p-maximum. Thus, $w(P \cap M)=w\left(P \cap M^{\prime}\right)$. Then, since $P \cap M^{\prime}$ contains more edges than $P \cap M, P \cap M$ contains one more thick edge than $P \cap M^{\prime}$. But then, $M^{\prime} \oplus P$ has weight $\nu(G)$ but $\left|M_{2}^{\prime}\right|<\nu\left(G_{2}\right)$, contradicting that $M^{\prime}$ is p-maximum. Thus, $P$ ends at $u$. Now, consider $P^{\prime}=P \cup\left\{u v^{\prime}\right\}$. If $w\left(P^{\prime} \backslash M^{\prime}\right)>w\left(P^{\prime} \cap M^{\prime}\right)$, then $w\left(P^{\prime} \oplus M^{\prime}\right)>w\left(M^{\prime}\right)$, which contradicts that $M^{\prime}$ is p-maximum. Thus, $w\left(P^{\prime} \backslash M^{\prime}\right) \leq w\left(P^{\prime} \cap M^{\prime}\right)$. Suppose $w\left(P^{\prime} \backslash M^{\prime}\right)<w\left(P^{\prime} \cap M^{\prime}\right)$. Let $x$ be the vertex such that $u x \in M^{\prime}$. Note that, since $u \in A_{1}(G), w(u x)=w\left(u v^{\prime}\right)=1$. Define $P^{\prime \prime}=P^{\prime} \backslash\left\{x u, u v^{\prime}\right\}$. Since $w\left(P^{\prime} \backslash M^{\prime}\right)<w\left(P^{\prime} \cap M^{\prime}\right)$, we have $w\left(P^{\prime \prime} \backslash M^{\prime}\right)<w\left(P^{\prime \prime} \cap M^{\prime}\right)$. But then, $P^{\prime \prime} \oplus M$ is a matching in $G-u$, and moreover $w\left(P^{\prime \prime} \oplus M\right)>w(M)$, contradicting that $M$ is p-maximum. Thus, $w\left(P^{\prime} \backslash M^{\prime}\right)=w\left(P^{\prime} \cap M^{\prime}\right)$. But then, $M^{\prime} \oplus P^{\prime}$ is a matching in $G$. Moreover, since $P^{\prime}$ is an even-length path and $w\left(M^{\prime} \oplus P^{\prime}\right)=w\left(M^{\prime}\right)$, the matchings $M^{\prime} \oplus P^{\prime}$ and $M^{\prime}$ have the same number of thick edges. Thus, $M^{\prime} \oplus P^{\prime}$ is a p-maximum matching in $G$. Since $M^{\prime} \oplus P^{\prime}$ does not cover $v, v \in D(G)$.

Let $v \in D(G)$. Let $M$ be a p-maximum matching in $G$ missing $v$. Then, since $M-u$ is p-maximum in $G-u$ and misses $v, v \in D(G-u)$.

(ii) First, we show that $D_{1}(G) \subseteq D_{1}(G-u)$. Let $v \in D_{1}(G)$ and $M$ be a p-maximum matching missing $v$. Let $M-u=M \backslash\{u x\}$ where $u x \in M$. Since the weight of $M-u$ is $w(M)-1$ and $u \in A_{1}(G)$, the matching $M-u$ is a p-maximum matching in $G-u$. Since $M-u$ misses $v, v \in D(G-u)$. Since, by Lemma $2, v \in D_{1}(G)$ implies that the edges incident with $v$ are thin, $v \notin D_{1,2}(G-u)$. Thus, $v \in D_{1}(G-u)$.

Now, we show that $D_{1}(G-u) \subseteq D_{1}(G)$. Let $v \in D_{1}(G-u)$. Note that $D_{1}(G-u) \subseteq D(G-u) \subseteq$ 
$D(G)$, where the first inclusion follows from the definition of the sets $D_{1}(G-u)$ and $D(G-u)$, the second inclusion by part (i). Thus, $v \in D(G)$. Suppose that there exists a p-maximum matching in $G$, which covers $v$ by a thick edge. Since $u \in A_{1}(G)$, this p-maximum matching does not contain $u v$. By removing the edge that covers $u$, a p-maximum matching in $G-u$ is obtained, which covers $v$ by a thick edge. But, since $v \in D_{1}(G-u)$, it is a contradiction. Thus, $v \notin D_{1,2}(G) \cup D_{2}(G)$. Since $v \in D(G)$ and $v \notin D_{1,2}(G) \cup D_{2}(G)$, this implies that $v \in D_{1}(G)$.

Lemma 4 Let $G$ be any graph. Let $u \in A_{1}(G)$. Then,

(i) $D_{1,2}(G-u) \subseteq D_{1,2}(G)$,

(ii) $D_{1,2}(G) \backslash D_{1,2}(G-u) \subseteq D_{2}(G-u)$.

(iii) Moreover, for each $v \in D_{1,2}(G) \backslash D_{1,2}(G-u)$, the only vertex incident to $v$ in $A_{1}(G)$ is $u$.

Proof. (i) First note that, $D_{1,2}(G-u) \subseteq D(G-u)=D(G)$, where the inclusion is by definition of the sets $D_{1,2}(G-u)$ and $D(G-u)$, and the equality is by Lemma 3(i). Thus, by the fact that, for each graph $G^{\prime}$ the sets $D_{1}\left(G^{\prime}\right), D_{2}\left(G^{\prime}\right)$ and $D_{1,2}\left(G^{\prime}\right)$ partition the set $D\left(G^{\prime}\right)$, we have $D_{1,2}(G-u) \subseteq D_{1}(G) \cup D_{2}(G) \cup D_{1,2}(G)$. Thus, Lemma 3(ii) (together with the fact that the sets $D_{1,2}(G-u)$ and $D_{1}(G-u)$ are disjoint) implies that $D_{1,2}(G-u) \subseteq D_{1,2}(G) \cup D_{2}(G)$. Thus, to prove that $D_{1,2}(G-u) \subseteq D_{1,2}(G)$, it is sufficient to show that, for each $v \in D_{1,2}(G-u)$, there exists a p-maximum matching in $G$, which covers $v$ by a thin edge. Let $v \in D_{1,2}(G-u)$ and $M$ be a p-maximum matching in $G-u$, which covers $v$ by a thin edge. Since $v \in D_{1,2}(G) \cup D_{2}(G)$, there exists a p-maximum matching, $M^{\prime}$ in $G$, which misses $v$. By definition of $A_{1}(G), u$ is covered by a thin edge, say $u x$, in $G$. Since $u \in A_{1}(G), M^{\prime}-u$ is a p-maximum matching in $G-u$. Moreover, the matching $M^{\prime}-u$ misses $v$. Consider the set $M \cup\left(M^{\prime}-u\right)$. It contains an alternating path $P$, starting at $v$ with an edge of $M$. Since $M$ and $M^{\prime}-u$ are p-maximum, this path is an even-length path containing the same number of thick edges of $M$ and $M^{\prime}-u$. Also, since the matching $M^{\prime}-u$ misses $x$, and the path ends with an edge of $M^{\prime}-u$, the path $P$ does not cover the vertex $x$. But then, the matching $\left(M^{\prime}-u\right) \oplus P$ is a p-maximum matching 
in $G-u$, which covers $v$ by a thin edge. Since this last matching does not cover the vertices $u$ and $x$, the matching $M^{\prime} \oplus P$ is a p-maximum matching in $G$ and it covers $v$ by a thin edge as well. Thus, $v \in D_{1,2}(G){ }^{24}$

(ii) Let $v \in D_{1,2}(G) \backslash D_{1,2}(G-u)$. Since the set $D_{1,2}(G)$ is nonempty by assumption (otherwise the result follows trivially), by definition of this set, there are two p-maximum matchings in $G$, say $M$ and $M^{\prime}$, where $M$ misses $v$ and $M^{\prime}$ covers $v$ by a thick edge. Since $u \in A_{1}(G)$, both $M-u$ and $M^{\prime}-u$ are p-maximum in $G-u$. By Lemma $3, v \notin D_{1}(G-u)$. Thus, $v \in D_{2}(G-u)$.

(iii) Let $v \in D_{1,2}(G) \backslash D_{1,2}(G-u)$. Let $M$ be a p-maximum matching in $G$ missing $v$. Suppose $v$ is incident to $v^{\prime} \in A_{1}(G)$ with $v^{\prime} \neq u$. Suppose $M$ contains $u v^{\prime}$. But then, since the matching $\left(M \backslash\left\{u v^{\prime}\right\}\right) \cup\{u v\}$ (which is obtained from $M$ by replacing the edge $u v^{\prime}$ with $u v)$ is also p-maximum and misses $v^{\prime}$, this contradicts with $v^{\prime} \in A_{1}(G)$. Thus, $M$ does not contain $u v^{\prime}$. Thus, $M-u$ covers $v^{\prime}$ by a thin edge, say $x v^{\prime}$ and misses $v$. If the edge $v v^{\prime}$ is thick, then $\left(M \backslash\left\{x v^{\prime}\right\}\right) \cup\left\{v v^{\prime}\right\}$ is a matching in $G-u$, and has a greater weight than $M-u$, contradicting that in the subgraph $G-u, M-u$ is p-maximum. If the edge $v v^{\prime}$ is thin, then $\left(M \backslash\left\{x v^{\prime}\right\}\right) \cup\left\{v v^{\prime}\right\}$ is also p-maximum in $G-u$, implying $v \notin D_{2}(G-u)$. But this contradicts part (ii) above.

Lemma 5 Let $G$ be any graph. Let $u \in A_{1}(G)$. Then, $A_{1}(G) \backslash\{u\}=A_{1}(G-u)$.

Proof. Let $v \in A_{1}(G) \backslash\{u\}$. Suppose that in each p-maximum matching in $G, u$ is matched to $v$. Let $v^{\prime}$ be a vertex in $D_{1}(G) \cup D_{1,2}(G)$, which is incident to $v$. Consider a p-maximum matching $M^{\prime}$ missing $v^{\prime}$. Since $u v \in M^{\prime}$, the matching $\left(M^{\prime} \backslash\{u v\}\right) \cup\left\{v v^{\prime}\right\}$ is also p-maximum in $G$ and misses $u$, which contradicts $u \in A_{1}(G)$. Thus, there exists at least one p-maximum matching in $G$ such that $u$ and $v$ are not matched. Let $M$ be such a p-maximum matching in $G$ and consider the matching $M-u$ in $G-u$. Since $u \in A_{1}(G)$ and $w(M-u)=w(M)-1$, the matching $M-u$ is p-maximum in $G-u$. Moreover, it covers $v$ by a thin edge. Thus,

\footnotetext{
${ }^{24}$ Note that in general, $D_{1,2}(G) \nsubseteq D_{1,2}(G-u)$. This can be seen via a simple example: Let $G=(V, E, w)$ be a weighted graph such that $V=\left\{v_{1}, v_{2}, v_{3}, v_{4}, v_{5}\right\}$ where $v_{1} v_{2}, v_{2} v_{3}$ are thick edges and $v_{3} v_{4}, v_{4} v_{5}$ are thin edges. Here, $D_{1,2}(G)=\left\{v_{3}\right\}, A_{1}(G)=\left\{v_{4}\right\}, D_{1}(G)=\left\{v_{5}\right\}$, and $D_{1,2}\left(G-v_{4}\right)=\emptyset$.
} 
$v \in D_{1}(G-u) \cup D_{1,2}(G-u) \cup A_{1}(G-u) \cup A_{1,2}(G-u) \cup C_{1}(G-u) \cup C_{1,2}(G-u)$. By Lemma 3 and 4, $v \notin D_{1}(G-u) \cup D_{1,2}(G-u)$. Suppose $v \in C_{1}(G-u) \cup C_{1,2}(G-u)$. By Lemma 3 and 4 , this is possible only if the set of neighbors of $v$ in $D_{1}(G) \cup D_{1,2}(G)$, say $V^{\prime}$, is in $D_{1,2}(G) \backslash D_{1,2}(G-u)$. But, Lemma 4 (iii) implies that, any vertex in $V^{\prime}$ has only one incident vertex in $A_{1}(G)$, which is $u$. Thus, $v \notin C_{1}(G-u) \cup C_{1,2}(G-u)$. Thus, $A_{1}(G) \backslash\{u\} \subseteq A_{1}(G-u) \cup A_{1,2}(G-u)$. Now, suppose that $v \in A_{1,2}(G-u)$. Let $M$ be a p-maximum matching in $G-u$ covering $v$ by a thick edge. Let $y \in D_{1}(G) \cup D_{1,2}(G)$ be a neighbor of $u$ and $M^{\prime}$ be a p-maximum matching in $G$, which is missing $y$. Also, let $u x \in M^{\prime}$. Note that $x \neq v$, since otherwise, $\left(M^{\prime} \backslash\{u v\}\right) \cup\{u y\}$ has the same weight as $M^{\prime}$, thus it is p-maximum in $G$ and missing $v$, which contradicts $v \in A_{1}(G)$. The matching $M^{\prime} \backslash\{u x\}$ is p-maximum in $G-u$. If $M$ misses $y$, then $M \cup\{u y\}$ is p-maximum and it covers $v$ by a thick edge, which contradicts $v \in A_{1}(G)$. Thus, $M$ covers $y$. Then, $M \oplus\left(M^{\prime} \backslash\{u v\}\right)$ contains a path $P$ starting at $y$ with an edge of $M$. Suppose the path $P$ covers $x$. Then, since $M^{\prime} \backslash\{u x\}$ misses $v, P$ should end at $x$ with an edge of $M$, which, by Lemma 1 , contradicts that in the subgraph $G-u, M^{\prime} \backslash\{u x\}$ is p-maximum. Thus, the path $P$ does not cover $x$. But then, $M^{\prime} \oplus P$ is p-maximum in $G$, covering $v$ by a thick edge, which contradicts $v \in A_{1}(G)$. Thus, $v \notin A_{1,2}(G-u)$. Thus, $A_{1}(G)-\{u\} \subseteq A_{1}(G-u)$.

Now, we show that $A_{1}(G-u) \subseteq A_{1}(G) \backslash\{u\}$. Suppose this is not true. Let $x \neq u$ such that $x \in A_{1}(G-u)$ but $x \notin A_{1}(G)$. Since for each p-maximum matching $M$ missing a vertex $x \neq u, M-u$ is also missing $x$ and p-maximum in $G-u$, each p-maximum matching in $G$ covers $x$. Similarly, since for each p-maximum matching $M$ covering a vertex $x \neq u$ by a thick edge, $M-u$ also covers $x$ by a thick edge and is p-maximum in $G-u$, no p-maximum matching in $G$ covers $x$ by a thick edge. Thus, $x \in A_{1}(G) \cup C_{1}(G)$. Suppose $x \in C_{1}(G)$. Then, by definition of $C_{1}(G), x$ is not incident to any vertex in $D_{1}(G) \cup D_{1,2}(G)$. But then, since by Lemma 3 and $4, D_{1}(G-u)=D_{1}(G)$ and $D_{1,2}(G-u) \subseteq D_{1,2}(G), x$ is not incident to any vertex in $D_{1}(G-u) \cup D_{1,2}(G-u)$ neither. But this contradicts $x \in A_{1}(G-u)$. Thus, $A_{1}(G-u) \subseteq A_{1}(G) \backslash\{u\}$.

Lemma 6 Let $u \in A_{1}(G)$. Then, in each p-maximum matching, $u$ is matched with a vertex 
in $D(G)$.

Proof. Let $u \in A_{1}(G)$ and $M$ be a p-maximum matching. Suppose $M$ contains $u v$ where $v \in A_{1}(G)$. Since $u \in A_{1}(G)$, the matching $M \backslash\{u v\}$ is p-maximum in $G-u$. By Lemma 5 , $v \in A_{1}(G-u)$. But, this contradicts that $M \backslash\{u v\}$ is p-maximum and misses $v$ in $G-u$. Now, suppose $M$ contains $u v$ where $v \in C_{1}(G) \cup C_{1,2}(G) \cup A_{1,2}(G)$. Let $v^{\prime} \in D_{1}(G) \cup D_{1,2}(G)$ be a vertex incident with $u$. The matching $M$ covers $v^{\prime}$, since otherwise, $(M \backslash\{u v\}) \cup\left\{u v^{\prime}\right\}$ is p-maximum in $G$ and misses $v$, contradicting $v \in C_{1}(G)$. Since $v^{\prime} \in D_{1}(G) \cup D_{1,2}(G)$, there exists a p-maximum matching missing $v^{\prime}$. Let $M^{\prime}$ be such a matching. The set $M \oplus M^{\prime}$ contains alternating paths and cycles. Since $v^{\prime}$ is covered by $M$ and missed by $M^{\prime}, M \oplus M^{\prime}$ contains an alternating path, $P$, starting at $v^{\prime}$. By Lemma 1 , that $M$ and $M^{\prime}$ are p-maximum implies that $P$ is not an augmenting path. Thus, the matchings $M \oplus P$ and $M^{\prime} \oplus P$ are p-maximum in $G$. There are two cases to consider.

Case 1: The path $P$ does not contain $u v$. Then, the matching $M \oplus P$ contains $u v$ and misses the vertex $v^{\prime}$. But then, since both $u v$ and $u v^{\prime}$ are thin edges, the matching $((M \oplus P) \backslash\{u v\}) \cup\left\{u v^{\prime}\right\}$ is p-maximum and missing $v$, which contradicts $v \in C_{1}(G) \cup C_{1,2}(G) \cup A_{1,2}(G)$.

Case 2: The path $P$ contains $u v$. Then, the matching $M^{\prime} \oplus P$ is p-maximum and contains $u v$. Since $u \in A_{1}(G)$, the matching $M \backslash\{u v\}$ is p-maximum in $G-u$. Also, it misses $v$. Thus, $v \in D(G-u)$. But, by Lemma 3(i), this contradicts $v \in C_{1}(G) \cup C_{1,2}(G) \cup A_{1,2}(G)$. Thus, $u$ is matched with a vertex in $D_{1}(G) \cup D_{1,2}(G) \subseteq D(G)$.

The last lemma completes the proof of part 1 of Theorem 1 . The second part of the theorem relies on a trivial extension of the Gallai's Lemma (Lovász and Plummer [10]); for the sake of completeness, we present the proof of this extension below.

Lemma 7 The components of $G-A_{1}(G)$ in $D(G)$, if any, are hypomatchable. Also, each p-maximum matching of $G$ contains a near-perfect matching of each such component.

Proof. By Lemma 3(i) and Lemma 5, the subgraph $G-A_{1}(G)$ is such that if we remove all the vertices in $A_{1}(G)$ one-by-one, $D\left(G-A_{1}(G)\right)=D(G)$. Let $M$ be a p-maximum matching in $G$. Let $H$ be a component of $G-A_{1}(G)$ in $D(G)$. By Lemma 6, the matching $M \backslash\left\{u x: u \in A_{1}(G)\right\}$ 
is p-maximum in $G-A_{1}(G)$. Now, consider the graph $G-A_{1}(G)$. Suppose that there are two p-maximum matchings $M^{\prime}$ and $M^{\prime \prime}$ missing the vertices $A$ and $B$ respectively, such that $|A|<|B|$. Now, suppose that all the vertices in $B \backslash A$ are covered by $M^{\prime}$. Let $u \in B \backslash A$. Since $M^{\prime \prime}$ misses $u$, the set $M^{\prime} \oplus M^{\prime \prime}$ contains a path $P$ starting at $u$ with an edge of $M^{\prime}$. Since both $M^{\prime}$ and $M^{\prime \prime}$ are p-maximum, Lemma 1 implies that the path $P$ is even. Also, since $|A \backslash B|<|B \backslash A|$, without loss of generality, we can assume that $P$ does not end in $A$. Note that $P$ does not contain any vertex of $A$. Then, by Lemma $1, M^{\prime} \oplus P$ is p-maximum and misses the vertices in $A \cup\{u\}$. Thus, there exists a p-maximum matching which misses $A$ and at least one vertex of $B \backslash A$. Now define the binary relation $\sim$ as follows: $u \sim v$ if and only if $u=v$ or no p-maximum matching misses both $u$ and $v$. Suppose $u \sim v$ and $v \sim w$. Let $M^{\prime}$ be a p-maximum matching missing $v$ and $M^{\prime \prime}$ missing $u$ and $w$. But then, there is a p-maximum matching missing $v$ and $w$, which is a contradiction. Thus, $u \sim w$ and $\sim$ is an equivalence relation. Now, since $H$ is connected, any two vertices of $H$ must be equivalent. Thus, any p-maximum matching misses at most one vertex of $H$. Also, since any vertex $u \in H$ is also in $D\left(G-A_{1}(G)\right), \nu\left(G-A_{1}(G)\right)=\nu\left(G-A_{1}(G)-u\right)$. Thus, the reduced submatching of $M$ on $H$ is a near-perfect matching of $H$.

\subsection{Gallai-Edmonds Decomposition Theorem}

Let $G=(V, E)$ be an undirected graph. ${ }^{25}$ The Gallai-Edmonds Decomposition partitions $V$ into three sets: $D(G)$ is the set of all vertices $v$ such that there is some maximum matching that leaves $v$ unmatched, $A(G)$ is the neighbor set of $D(G)$, and $C(G)$ is the set of all remaining vertices.

Corollary 1 (Gallai-Edmonds Decomposition Theorem) Given a graph $G=(V, E), D(G)$ consists of odd components of $G-A(G)$ such that each such component is hypomatchable and

${ }^{25}$ Note that this is a particular case of the graph introduced in the Subsection 5.1; while the subsections 5.1 and 5.2 are on a particular class of weighted graphs, here we focus on non-weighted graphs (i.e. each edge has weight one). This implies that the definition of p-maximum matching becomes vacuous; thus in what follows, we refer to maximum (cardinality) matchings rather than p-maximum matchings. For the definition of a p-maximum or maximum (cardinality) matching, please see Section 3. 
$C(G)$ consists of even components of $G-A(G)$ such that each such component has a perfect matching. In each maximum matching of $G=(V, E)$,

(i) each vertex in $A(G)$ is matched to a vertex in $D(G)$,

(ii) each even component of $G-A(G)$ has a perfect matching,

(iii) each odd component of $G-A(G)$ has a near-perfect matching such that only one vertex is either unmatched or matched to a vertex in $A(G)$.

To see how the GED Theorem is implied by Theorem 1, first note that the GED Theorem is on the set of non-weighted graphs. Thus, it corresponds to a special case of our model where for any two pairs $x, y \in N, r_{x, y} \in\{0,1\}$.

Let $(N, R)$ be a problem and $G=(V, E)$ be its graphical representation as defined in the Subsection 5.1, with each edge having weight one (thus the function $w$ is redundant and excluded from the representation). Since by definition of this representation, $V=N$, we have that $D(N, R)=D(G)$. Also, since there are only PLE's, $A_{1}(N, R)=A(G)$. Thus, part (i) of the GED Theorem follows directly from part (1) of Theorem 1. Note that, by definitions of $A_{1}(N, R)$ and $D(N, R)$, for each $x \in D(N, R)$ and $y \in N \backslash A_{1}(N, R), x$ is not compatible with $y$ and $y$ is not compatible with $x$. Thus, any component of $N \backslash A_{1}(N, R)$ is either in $D(N, R)$ or in $N \backslash\left(D(N, R) \cup A_{1}(N, R)\right) .{ }^{26}$ Thus, part 2 (a) of Theorem 1 implies that any component of $D(G)(=D(N, R))$ is hypomatchable (and therefore odd). Also, since in each maximum matching, each vertex in $A(G)$ is matched to a vertex in $D(G)$ and each vertex in $C(G)$ is matched, each component of $C(G)$ must be even and have a perfect matching. Finally, parts 2(b) and 2(c) of Theorem 1 imply part (iii) of the GED Theorem.

\subsubsection{Fractional matchings}

Another extension of the GED Theorem is in the context of fractional matchings: Given a graph $G=(V, E)$, a fractional matching is an assignment of the values $0, \frac{1}{2}, 1$ to the edges $E$ in such a way that for each vertex, the sum of the values on the incident edges is at most 1

\footnotetext{
${ }^{26}$ Note that this does not hold in our model in general.
} 
(Mühlbacher et al. [11], Pulleyblank [14]). A fractional matching is called basic if all the edges having value $\frac{1}{2}$ form vertex-disjoint odd cycles. A basic fractional matching is called a U-matching if it is a maximum fractional matching for which the number of edges in the odd cycles is minimum. The characterization of the U-matchings is derived from the GED Theorem (Pulleybank [14]). Seemingly, this model of fractional matchings has similarities to our model of kidney exchange, but it is essentially different and our main result (Theorem 1) is independent from the characterization of the U-matchings (Theorem 4, Pulleybank [14].) There are two main differences: first, our model relies on the non-homogeneous weights of the edges as opposed to the uniform edges; second, in a fractional matching, there can be two edges which are incident with the same vertex, whereas in our model, a matching is a subset of the edges such that no two edges meet at a common vertex. Due to these differences, the two characterization results are quite separate from each other. While the characterization of the U-matchings is built on the partition of the GED Decomposition, i.e. on the partition of $V$ into the sets $D(G), A(G)$ and $C(G)$, the characterization of the p-matchings in our model is obtained via a completely different partition of the set $V$. Actually, the structure of the U-matchings coincides with the parts (i) and (ii) of the GED Theorem. The only difference from the GED Theorem is that a U-matching may induce a perfect fractional matching for some odd component of $G-A(G)$, which means that in this U-matching, there is an odd cycle such that each of its edges has value $\frac{1}{2}$. (Theorem 4, Pulleybank [14]). On the other hand, our main result gives a different and more restricted structure on a more complex partition of $V$, yet this characterization is rich enough to include the GED structure as a special case. 


\section{REFERENCES}

1. B. Ata, A. Skaro and S. Tayur, OrganJet: overcoming geographical disparities in access to deceased donor kidneys in the United States, (2012), mimeo.

2. A. Bogomolnaia and H. Moulin, Random matching under dichotomous preferences, Econometrica 72 (2004), 257-279.

3. F. L. Delmonico, Exchanging kidneys advances in living-donor transplantation, The New England Journal of Medicine 350 (2004), 1812-1814.

4. F. L. Delmonico, G. S. Lipkowitz, P. E. Morrissey, J. S. Stoff, J. Himmelfarb, W. Harmon, M. Pavlakis, H. Mah, J. Goguen, R. Luskin, E. Milford, G. B. M. Chobanian, B. Bouthot, M. Lorber, and R. J. Rohrer, Donor kidney exchanges, American Journal of Transplantation 4 (2004), 1628-1634.

5. J. Edmonds, Paths, trees, and flowers, Canadian Journal of Mathematics 17 (1965), 449467.

6. T. Gallai, Kritische Graphen II, Magyar Tudományos Akadémia- Matematikai Kutató Intézetének Közleményei 9 (1964), 401-413.

7. T. Gallai, Maximale Systeme Unabhängiger Kanten, Magyar Tudományos AkadémiaMatematikai Kutató Intézetének Közleményei 8 (1963), 373-395.

8. S. E. Gentry, Dorry L. Segev, and R. A. Montgomery, A comparison of populations served by kidney paired donation and list paired donation, American Journal of Transplantation, 5 (2005), 1914-1921.

9. I. Kaplan, J. A. Houp, M. S. Leffell, J. M. Hart, and A. A. Zachary, A computer match program for paired and unconventional kidney exchanges, American Journal of Transplantation 5 (2005), 2306-2308. 
10. L. Lovász and M. D. Plummer, Matching Theory, North-Holland Press, New York, 1986.

11. J. R. Mühlbacher, F. X. Steinparz, and G. Tinhofer, On certain classes of fractional matchings, Discrete Applied Mathematics, 9 (1984) 235-244.

12. A. Nicolò and C. Rodriguez-Álvarez, Transplant quality and patients preferences in paired kidney exchange, Games and Economic Behavior 74 (2011), 299-310.

13. A. Nicolò and C. Rodriguez-Álvarez, Age-based preferences: incorporating compatible pairs into paired kidney exchange, (2011), mimeo.

14. W. R. Pulleyblank, Fractional matchings and the Edmonds-Gallai Theorem, Discrete Applied Mathematics 16 (1987), 51-58.

15. F. T. Rapaport, The case for a living emotionally related international kidney donor exchange registry, Transplantation Proceedings 18 (1986), 5-9.

16. L. F. Ross, , D. T. Rubin, M. Siegler, M. A. Josephson, J. R. Thistlethwaite, Jr. and E. S. Woodle, Ethics of a paired kidney exchange program, The New England Journal of Medicine 336 (1997), 1752-1755.

17. L. F. Ross and E. S. Woodle, Ethical issues in increasing living kidney donations by expanding kidney paired exchange programs, Transplantation 69 (2000), 1539-1543.

18. A. E. Roth, T. Sönmez, and M.U. Ünver, Kidney exchange, Quarterly Journal of Economics 119 (2004), 457-488.

19. A. E. Roth, T. Sönmez, and M.U. Ünver, Pairwise kidney Exchange, Journal of Economic Theory 125 (2005), 151-188.

20. A. E. Roth, T. Sönmez, and M.U. Ünver, A kidney exchange clearinghouse in New England, American Economic Review: Pap. Proc. 95 (2005), 376380. 
21. A. E. Roth, T. Sönmez, M.U. Ünver, Utilizing list exchange and nondirected donation through chain paired kidney donations, American Journal of Transplantation 6 (2006), 2694-2705.

22. A. E. Roth, T. Sönmez, and M.U. Ünver, Efficient Kidney exchange: coincidence of wants in markets with compatibility-based preferences, American Economic Review, 97 (2007), 828-851.

23. D. L. Segev, S. E. Gentry, J. K. Melancon, R. A. Montgomery, Characterization of waiting times in a simulation of kidney paired donation, American Journal of Transplantation 5 (2005), 2448-2455.

24. D. L. Segev, S. E. Gentry, D. S. Warren, B. Rech, R. A. Montgomery, Kidney paired donation and optimizing the use of living donor organs, Journal of American Medical Association 293 (2005), 1883-1890.

25. T. Sönmez and M.U. Ünver, Altruistically unbalanced kidney exchange, (2011), mimeo.

26. M.U. Ünver, Dynamic kidney exchange, Review of Economic Studies 77 (2010), 372-414.

27. Ö. Yllmaz, Kidney exchange: an egalitarian mechanism, Journal of Economic Theory 146 (2011), 592-618.

28. S. A. Zenios, Optimal control of a paired-kidney exchange program, Management Science 48 (2002), 328-342. 